\title{
Neumonitis por hipersensibilidad en la ciudad de México
}

José G. C arrillo-Rodríguez, M.C., ${ }^{(1)}$ Raúl H. Sansores, M. en C., ${ }^{(1)}$ A mérica C astrejón, Téc. en T.S., ${ }^{(1)}$ Rogelio Pérez-Padilla, M.C., ${ }^{(2)}$ A lejandra Ramírez-Venegas, M.C., M. en C., ${ }^{(1)}$ Mo isés Selman, M. en C. ${ }^{(3)}$

\section{Carrillo-Rodríguez JG, Sansores RH, Castrejón A, Pérez-Padilla $R$, Ramírez-Venegas A, Selman $M$. Neumonitis por hipersensibilidad en la ciudad de México. \\ Salud Publica Mex 2000;42:201-207.}

\section{Resumen}

Objetivo. Determinar la asociación entre la zona urbana de origen del paciente en la ciudad de México y la prevalencia de neumonitis por hipersensibilidad inducida por antígeno aviario. Material y métodos. Se trata de un estudio de casos y controles realizado en el Instituto $\mathrm{N}$ acional de Enfermedades Respiratorias, en la ciudad de México, en el año de 1999. Se estudiaron 109 casos con neumonitis por hipersensibilidad y 184 controles: de éstos, 39, con fibrosis pulmonar idiopática; 63, con tuberculosis pulmonar, y 82, con asma. La ciudad de México y las zonas conurbadas se dividieron en cinco zonas geo gráficas: centro, noreste, sureste, no roeste y el suroeste. Se calcularon las prevalencias de las diferentes enfermedades por zona urbana de los pacientes que participaron en el estudio; como medida de asociación, se estimó la razón de momios, con un intervalo de confianza al 95\%. A simismo, se realizó regresión logística múltiple ajustando por edad, sexo y estrato socio económico. Resultados 0 chenta casos de neumonitis por hipersensibilidad se concentraron en el sur del noreste de las zonas conurbadas y la parte norte del sureste de la ciudad de México, 48 y 32, respectivamente ( $R M=3.86$, IC 95\% 2.17-6.96). Treinta y seis controles de asma se localizaron en el suroeste de la ciudad de México, zona donde se ubica el Intituto $\mathrm{N}$ acional de Enfermedades Respiratorias $(p<0.05)$ y cuatro en la zona conurbada. Los controles de tuberculosis pulmonar y fibrosis pulmonar idio pática estuvieron dispersos en la ciudad de México y en las zonas conurbadas. Conclusiones. La zona sur del noreste y el

\author{
Carrillo-Rodríguez JG, Sansores RH, \\ Castrejón A, Pérez-Padilla R, \\ Ramírez-Venegas A, Selman M. \\ Hypersensitivity pneumonitis \\ in Mexico City.
}

Salud Publica Mex 2000;42:201-207.

\begin{abstract}
A bstract
Objective. To investigate the association between the urban area of origin of patients and the prevalence of hypersensitivity pneumonitis (HP), induced by avian antigens. Material and methods A case-control study was conducted in 1999 at the National Institute of Respiratory D iseases (N IRD ). C ases were 109 consecutive HP patients and controls were 184 patients: 39 with idiopathic pulmonary fibrosis (IPF), 63 with pulmonary tuberculosis (PTB), and 82 with asthma. Mexico $C$ ity and surrounding counties (SC) were divided into 5 geographical areas: 1) Downtown; 2) N orth-East (N E);3) South-East (SE);4) N orth-W est (N W ) and 5) South-W est (SW ). Statistical analysis consisted of calculation of disease prevalence by urban area; associations were assessed with odds ratios and $95 \%$ confidence intervals. Multivariate analysis with multiple logistic regression was performed to adjust for age, gender and socioeconomic level. Results. Eighty HP cases were located in the NE southernmost and SE northernmost areas of Mexico City (48 and 32, respectively) $(0 \mathrm{R}=3.86 ; 95 \% \mathrm{Cl}$ 2.17-6.96). Thirty-six controls with asthma came from the SW area, (where NIRD is located) $(p<0.05)$, and four from $S C$. Controls with PTB and IPF were scattered throughout the study area. Conclusions.The N E southernmost and SE northernmost areas were associated with HP. The cause of HP may not be geographical; a garbage dump used to be located in this area, suggesting that exposure to organic particles might contribute to the development of HP in susceptible individuals.
\end{abstract}

(1) Clínica de Enfermedades Intersticiales del Pulmón, Instituto N acional de Enfermedades Respiratorias (IN ER), México.
(2) Subdirección General Médica, IN ER, México.
(3) Subdireccion General de Investigación, IN ER, México.

Fecha de recibido: 27 de agosto de 1999 - Fecha de aceptado: 03 de abril de 2000

Solicitud de sobretiros: Dr. José G uillermo Carrillo Rodríguez. Instituto N acional de Enfermedades Respiratorias, Secretaría de Salud. Calzada de Tlalpan 4502, 14080 México, D.F., México.

Correo electrónico: josecr@ data.net.mx 
norte de la sureste están asociadas a la neumonitis por hipersensibilidad. Las causas de esta asociación no parece ser geográfica, pero existe el antecedente de que esa zona fue basurero de la ciudad, por lo que partículas orgánicas en el ambiente pudieran coadyuvar a la aparición de esta enfermedad.

Palabras clave: pulmón de criadores de aves; zonas urbanas; partículas orgánicas; México
Key words: bird fancier's lung; urban zones; organic particles; Mexico
$\mathrm{L}$ a inhalación y exposición sostenida de polvos puede condicionar, en algunas personas susceptibles, enfermedades de las vías aéreas y del parénquima pulmonar. Cuando los polvos son de origen orgánico el trastorno se conoce con el nombre de neumonitis por hipersensibilidad (NH). En el Instituto Nacional de Enfermedades Respiratorias (INER) la exposición a las aves, como palomas, es la causa más común. ${ }^{1-3}$ La enfermedad puede presentarse en forma aguda, subaguda o crónica, esta última es la que habitualmente observamos debido, por un lado, a la tardanza con la que los pacientes acuden a buscar ayuda médica y, por otro, a que la enfermedad no es fácilmente reconocida por los médicos no especializados. Esta entidad se caracteriza fundamentalmente por inflamación intersticial de predominio mononuclear en su fase crónica y que, dependiendo de muchos factores, la mayoría desconocidos, puede, en un porcentaje importante de los casos, desencadenar la fibrosis pulmonar con la consecuente invalidez física y muerte a mediano plazo. ${ }^{4,5}$ Esta progresión maligna de las $\mathrm{NH}$ es extremadamente rara en otros países, donde la evolución de los pacientes afectados suele ser benigna a pesar de tener estrecha convivencia con aves. ${ }^{6-8}$ Una de las múltiples diferencias posibles, entre los enfermos de nuestro país y los de otros países, es el ambiente en el que se desarrolla la enfermedad..$^{9,10}$

En la Clínica de Fibrosis Pulmonar del INER se le dedica especial atención a estos pacientes y en particular a todos los factores potencialmente involucrados en el curso de la enfermedad. La mayoría están incluidos en algún protocolo de estudio y requieren seguimiento y vigilancia permanentes. El objetivo del presente estudio fue determinar la asociación entre la zona urbana de origen del paciente en la ciudad de México y la prevalencia de neumonitis por hipersensibilidad inducida por antígeno aviario. Los hallazgos derivados de este estudio podrían tener impacto a diferentes niveles en la salud pública. Por ejemplo, la asociación entre la zona urbana de origen y la enfermedad podría deberse a factores ambientales ausentes en otros países y esto podría explicar, al menos par- cialmente, la tórpida evolución de esta enfermedad en México. Propiciaría, por lo tanto, líneas de investigación orientadas a determinar la influencia del ambiente en esta enfermedad y, finalmente, podrían surgir recomendaciones para limitar la convivencia con aves, al menos, en algunas zonas de la ciudad de México.

\section{Material y métodos}

Se realizó un estudio de casos y controles con base hospitalaria, provenientes de la Clínica de Fibrosis Pulmonar del INER. Después de tres años (1992-1994) de hacer visitas a domicilio diseñadas para conocer las características ambientales de la vivienda y eventualmente para seguir la evolución de los pacientes que dejan de asistir a su consulta, la trabajadora social se percató de que la mayor parte de las visitas domiciliarias se dirigían hacia una misma zona geográfica. Esto que fue sólo una observación durante las horas laborales, la convertimos en una hipótesis de trabajo mediante la cual postulamos que un porcentaje importante de pacientes con $\mathrm{NH}$ proviene de la misma zona urbana y que la zona de origen de los pacientes y la prevalencia de la enfermedad podría no ser casual; para comprobar esta hipótesis se llevó a cabo este estudio con 109 casos y 246 controles, en el año de 1999.

Casos. Se incluyeron 109 pacientes consecutivos con diagnóstico de $\mathrm{NH}$, mismo que se estableció de acuerdo con los criterios previamente establecidos, ${ }^{3}$ a saber: a) exposición a las aves con relación causa-efecto; b) disnea progresiva de esfuerzo; c) alivio de los síntomas al alejarse del antígeno agresor; d) anticuerpos séricos contra el antígeno; e) radiografía postero-anterior de tórax con imágenes nodulares o reticulonodulares; f) pruebas funcionales respiratorias con patrón restrictivo o predominantemente restrictivo, y g) espécimen histológico de pulmón obtenido de biopsia a cielo abierto con datos compatibles de $\mathrm{NH}$.

Controles. Se escogieron 246 pacientes consecutivos con diagnóstico de fibrosis pulmonar idiopática (FPI); ${ }^{5} 100$ pacientes con diagnóstico de tuberculosis 
pulmonar $^{11}$ y 100 pacientes con diagnóstico de asma bronquial. Los diagnósticos de cada una de las enfermedades se estableció de acuerdo con los criterios correspondientes internacionalmente aceptados. ${ }^{12}$ Del grupo seleccionado se excluyó del estudio a seis pacientes con NH; siete, con FPI; 37, con TBP, y a 18 enfermos de asma, por no vivir en el Estado de México o en la ciudad de México.

Zona urbana de origen de los pacientes (ZUOP). Para establecer la zona urbana de origen de cada paciente se tomó la dirección registrada en la hoja de trabajo social del expediente clínico. El mapa del Distrito Federal (DF) se dividió en cinco zonas geográficas, a saber: a) centro, que abarca la delegación Cuauhtémoc; b) zona noroeste (NE), que comprende las delegaciones Venustiano Carranza, Iztacalco, Gustavo A. Madero y parte de Iztapalapa, además incluye a las zonas conurbadas del Estado de México por su estrecha cercanía, éstas son: Los Reyes La Paz, Ciudad Netzahualcóyotl, Chalco, Texcoco y Chimalhuacán; c) zona sureste (SE), que abarca las delegaciones de Tláhuac, Tlalpan, Xochimilco, Milpa Alta y las zonas conurbadas mexiquenses; d) zona noroeste (NO), que comprende las delegaciones
Miguel Hidalgo, Azcapotzalco y las conurbadas del Estado de México como Tlalnepantla, Ciudad Satélite, Naucalpan, Ecatepec, Coacalco, Cuautitlán, y e) zona suroeste (SO), que contiene las delegaciones Magdalena Contreras, Alvaro Obregón y Coyoacán, y las zonas conurbadas correspondientes de Cuajimalpa y Huixquilucan (figura 1).

La clasificación socioeconómica a que cada paciente pertenecía se obtuvo del expediente, y se realizó con base en un tabulador existente en el Departamento de Trabajo Social, de acuerdo con el ingreso económico mensual aproximado, la vivienda y la alimentación de la familia. El estudio socioeconómico da lugar a siete estratos diferentes. Se consideró de nivel bajo a los que cayeron en los tres primeros números del tabulador (1x, 1 y 2). Para la NH hubo nueve pacientes de nivel socioeconómico alto; para la TBP, nueve; para FPI, cuatro, y para asma, 16, respectivamente. Para corroborar la dirección se hicieron 20 visitas seleccionadas al azar de cada uno de los grupos de estudio.

Se obtuvo la prevalencia de las diferentes enfermedades en las diversas zonas urbanas. Mediante un análisis de contingencia, usando tablas $2 \times 2$, se obtuvo

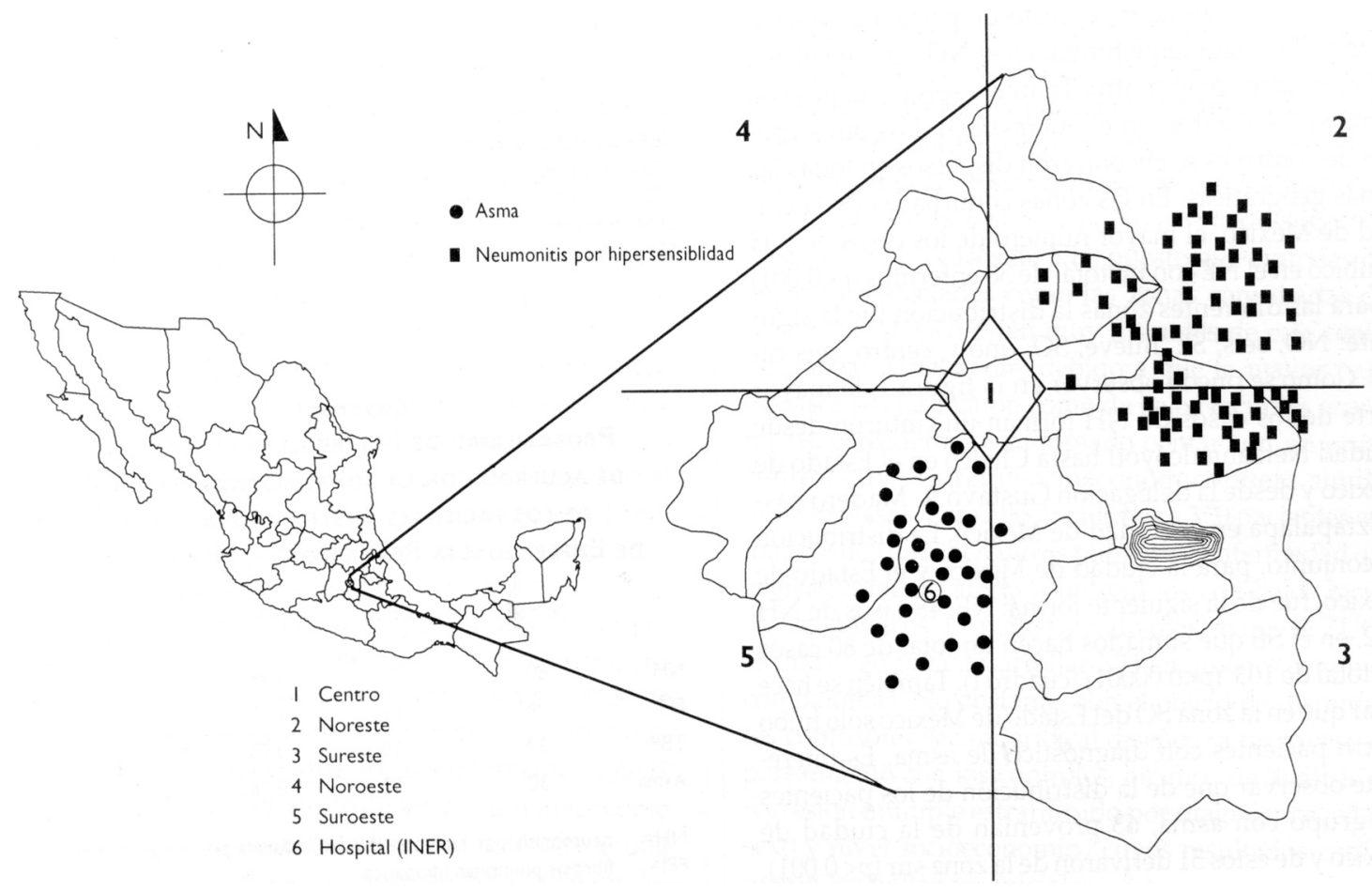

Figura 1. Sitios de A fLUENCIA DE LOS PACIENTES CON NEUMONITIS POR HIPERSEN SIBILIDAD Y DE LOS ENFERMOS ASMÁtICOS. Ciudad de México y zona conurbada (Estado de México). Instituto Nacional de Enfermedades Respiratorias, MÉxICO, 1999 
para cada enfermedad la razón de momios, con intervalo de confianza (IC) a 95\%. Se realizó un análisis de regresión logística múltiple para determinar la asociación entre ZUOP y NH ajustado por diferentes confusores como la edad, el sexo y el estrato socioeconómico.

\section{Resultados}

De los 109 casos con NH, 88 fueron mujeres y 21, hombres, con una edad promedio de $35 \pm 16$ años y $29 \pm 22$ años, respectivamente. De los pacientes con FPI, 32 correspondieron al sexo femenino ( $42 \pm 15$ años) y siete, al sexo masculino ( $44 \pm 16$ años). De los pacientes con TBP, 36 fueron del sexo femenino $(43 \pm 16$ años) y 27 , del sexo masculino (41 \pm 12 años). De los pacientes asmáticos, 60 fueron mujeres ( $43 \pm 15$ años) y 22 , hombres ( $39 \pm 15$ años). El Departamento de Trabajo Social los clasificó de acuerdo con su nivel socioeconómico en el estrato más bajo en todos los casos con excepción de nueve pacientes. Las visitas realizadas al azar coincidieron con los datos que proporcionaron los pacientes al momento de su registro. En la ciudad de México hubo 49 casos de NH; 10 pacientes fueron de la zona NE y 23, de la zona SE, el resto de pacientes se localizaron de la siguiente forma: en el NO, tres; en el SO, ocho, y cinco, en el centro. Treinta y seis pacientes con asma se localizaron en el SO $(p<0.05)$. Los otros grupos de controles se encontraron dispersos en todas las zonas geográficas. En las zonas conurbadas de la ciudad de México, el mayor número de los casos de $\mathrm{NH}$ se ubicó en el NE con un total de 38 enfermos $(p<0.001)$ y para las diferentes zonas la distribución fue la siguiente: NO, seis; SE, nueve; SO, uno y, centro, seis casos. Como se puede observar en la figura 1, la mayor parte de los casos de $\mathrm{NH}$ forman un cinturón desde Ciudad Netzahualcóyotl hasta Chalco en el Estado de México y desde la delegación Gustavo A. Madero hasta Iztapalapa en la ciudad de México. La distribución en conjunto, para la ciudad de México y el Estado de México, fue de la siguiente forma: $\mathrm{NE}, 48$ casos de $\mathrm{NH}$ y 32, en el SE que sumados hacen un total de 80 casos del total de 103, $(p<0.00001)$ (cuadro I). También se hace notar que en la zona SO del Estado de México sólo hubo cuatro pacientes con diagnóstico de asma. Es interesante observar que de la distribución de los pacientes del grupo con asma, 63 provenían de la ciudad de México y de éstos 51 derivaron de la zona sur $(p<0.001)$. Los pacientes con tuberculosis pulmonar estuvieron dispersos tanto en la ciudad de México como en el Estado de México.

La razón de momios (RM) para $\mathrm{NH}$ al vivir en la franja formada por el SE Y NE tanto de la ciudad de
México como del Estado de México fue estadísticamente significativa ( $\mathrm{RM}=3.8$, IC 95\% 2.17-6.96) (cuadro II). El análisis de regresión logística múltiple ajustado por sexo, edad y estrato socioeconómico confirmó esta asociación ( $\mathrm{RM}=2.86$, cuando se compara con FPI; 3.1 con TBP, y 0.29 con asma).

\section{Discusión}

Los resultados de este trabajo indican que la ZUOP y la prevalencia de la enfermedad en esa zona no es ca-

Cuadro I

Prevalencia de las diferentes enfermedades PULMONARES EN LAS ZONAS URBANAS DE ORIGEN DE LOS PACIENTES. In STITUTO N ACIONAL DE EN FERMEDADES ResPIRATORIAS, MÉXICO, 1999

\begin{tabular}{lrrrrrr} 
& NO & NE & SO & SE & Centro & Total \\
NH (\%) & $9(8.7)$ & $48(46.6)$ & $9(8.7)$ & $32(31.1)$ & $5(4.9)$ & 103 \\
\hline FPI (\%) & $5(12.8)$ & $13(33.3)$ & $8(20.5)$ & $11(28.2)$ & $2(5.1)$ & 39 \\
\hline TBP (\%) & $12(19)$ & $16(25.4)$ & $14(22.2)$ & $14(27)$ & $4(6.3)$ & 63 \\
\hline Asma (\%) & $12(14.6)$ & $10(12.2)$ & $36(43.9)$ & $20(24.4)$ & $4(4.9)$ & 82 \\
Total (\%) & $38(13.2)$ & $87(30.3)$ & $67(23.3)$ & $80(27.9)$ & $15(5.2)$ & 287
\end{tabular}

$\mathrm{NH}=$ neumonitis por hipersensibilidad inducida por antígeno aviario

$\mathrm{FPI}=$ fibrosis pulmonar idiopática

$\mathrm{TBP}=$ tuberculosis pulmonar

$\mathrm{N} \mathrm{O}=$ noroeste

$\mathrm{NE}=$ noreste

$\mathrm{SO}=$ suroeste

$\mathrm{SE}=$ sureste

\section{Cuadro II}

Probabilidad de desarrollar enfermedad

DE ACUERDO CON LA ZONA URBANA DE ORIGEN DE LOS PACIENTES. INSTITUTO N ACIONAL De Enfermedades Respiratorias, México, 1999

\begin{tabular}{lcccc} 
& NE+SE & Otras & RM & IC $95 \%$ \\
$\mathrm{NH}$ & 80 & 23 & 3.86 & $2.17-6.96$ \\
\hline FPI & 24 & 15 & 1.17 & $0.55-2.48$ \\
\hline TBP & 33 & 30 & 0.73 & $0.40-1.34$ \\
\hline Asma & 30 & 52 & 0.26 & $0.15-0.47$
\end{tabular}

$\mathrm{NH}=$ neumonitis por hipersensibilidad inducida por antígeno aviario $\mathrm{FPI}=$ fibrosis pulmonar idiopática

$\mathrm{TBP}=$ tuberculosis pulmonar

$N E+S E=l a$ suma de las zonas sureste y noreste

$\mathrm{RM}=$ razón de momios

0 tras $=$ Se refiere a las otras zonas geográficas urbanas (N O , SO, Centro y otras provincias del país) 
sual, sino que existe una significativa asociación entre ellas. De igual forma los resultados señalan que existe un riesgo aumentado (3.8 veces) de desarrollar $\mathrm{NH}$ en quienes tienen convivencia con aves, como las palomas, y viven en la franja que incluye el SE y NE, situación que no se observó para FPI. Los resultados también sugieren que el hecho de vivir en esta zona tiene un aparente "efecto protector" para tener asma o TBP.

Respecto de estos resultados es conveniente discutir acerca del sesgo de selección, así como de algunos confusores potenciales. El diseño de este trabajo seleccionó como casos a aquellos pacientes que tuvieran diagnóstico de $\mathrm{NH}$ y como controles a los que en forma incontrovertible no lo tuvieran. A cada grupo, en forma retrospectiva, se le determinó la zona urbana de origen. Por lo tanto, todos los pacientes tuvieron la misma posibilidad de venir de alguna zona urbana en particular. El sesgo de selección lo determina la posibilidad de que el grupo de pacientes con NH hubiera seleccionado a nuestro Instituto por al menos dos razones. Una, la cercanía y, la otra, el prestigio que el Instituto posee, entre la comunidad médica, en el conocimiento y tratamiento de este tipo de enfermedades. La confusión se podría originar de las características de los pacientes inherentes a la enfermedad. La mayor parte de los afectados por esta enfermedad son mujeres jóvenes de un estrato socioeconómico bajo. Así, el sexo, la edad y el nivel socioeconómico podrían actuar como confusores comunes a varios padecimientos y no exclusivamente a la $\mathrm{NH}$ y, por lo tanto, la admisión hospitalaria de cualquiera de ellos podría ser igualmente prevalente.

El grupo de pacientes con FPI y asma representan un buen control para eliminar el sesgo de selección y la popularidad, puesto que si éstos hubieran sido las causas por las que los pacientes con $\mathrm{NH}$ llegaron al Instituto, los pacientes con FPI y asma también debieron haber llegado de esas zonas. Los resultados muestran que lejos de ello, los pacientes con FPI vinieron de todas las zonas geográficas sin predominio de ninguna. En cambio, los pacientes con asma eligieron los centros hospitalarios de mayor cercanía a su lugar de residencia (figura 1). Es por ello que se observó que 43.9\% de los asmáticos que acudió al Instituto es de la zona del suroeste. Este efecto de selección aparece como un sesgo en el análisis estadístico que le ofrece una aparente protección a los vecinos del SE y SO para desarrrollar NH. Por el mismo sesgo, este efecto aparecería como riesgo para desarrollar asma entre los pacientes que viven en esa área geográfica si el análisis se hubiera abordado en esa dirección.
De los pacientes con $\mathrm{NH}$ en este estudio, $81 \%$ pertenecen al sexo femenino, provienen de un estrato socioeconómico bajo y su edad oscila entre los 15 y 66 años, con un promedio de 37 años. De acuerdo con estas características, escogimos tres grupos de pacientes como controles. Se incluyó a pacientes con fibrosis pulmonar idiopática con el objetivo de comparar pacientes con una enfermedad del intersticio pulmonar similar a la NH, pero con mecanismos patogénicos diferentes. Además, puesto que clínicamente esta enfermedad es indistinguible de la fibrosis pulmonar asociada a las $\mathrm{NH}$, si existiera para el Instituto una predilección de los médicos o instituciones que nos envían pacientes, ninguno de ellos podría diferenciar entre NH y FPI. Por lo tanto, un sesgo de selección en este sentido sería improbable. El grupo de asma se seleccionó para tener un grupo de pacientes predominantemente del sexo femenino (88 de 100) y cuya edad promedio fuera similar a los casos ( $37 \pm 13$ años). Asimismo, este grupo tiene la ventaja de representar un padecimiento que también tiene componentes patogénicos inmunológicos y ambientales.

Los casos de asma son un excelente grupo control para nuestra hipótesis, pues encontramos que del Estado de México sólo cuatro pacientes acudieron al INER, y de otras partes del país, 18. En cambio, de la zona sur acudieron 51 pacientes. Aunque esta asociación es significativa respecto al norte, se puede observar claramente un sesgo de selección condicionado por la cercanía a esta institución. No se puede sugerir lo mismo para los casos de $\mathrm{NH}$, puesto que tanto las delegaciones afectadas como las zonas conurbadas del Estado de México están muy distantes de este centro de trabajo. Finalmente, debido a que la mayor parte de casos se clasificaron como de bajos recursos económicos, consideramos apropiado contar con un grupo control cuyo estrato socioeconómico fuera similar al de los casos. Para ello, incluimos a 100 pacientes con diagnóstico de tuberculosis pulmonar, enfermedad que preferentemente afecta a individuos con bajos recursos económicos. El análisis crudo mostró que la ZUOP es un riesgo para desarrollar $\mathrm{NH}$ en quienes conviven con palomas. No obstante, con el objeto de disminuir los confusores secundarios al diseño o a un exceso de pareamiento con los controles, hicimos un análisis de regresión múltiple estratificado por diagnóstico, edad, sexo y nivel socioeconómico cuyos resultados confirmaron los hallazgos iniciales.

Por lo tanto, es improbable que nuestros resultados estén sesgados por la selección que los pacientes pudieron haber hecho o por efecto de confusores debido a que el análisis estadístico y el diseño de este 
trabajo propició la misma probabilidad para que cualquiera de las enfermedades estudiadas proviniera de cualquiera de las zonas de la ciudad de México o del Estado de México. Por lo que estos resultados permiten asumir que debe existir algún factor común en el ambiente que condiciona la aparición de la enfermedad en las personas susceptibles. En este sentido, vale la pena recordar que la zona de la delegación de Iztapalapa fue durante más de 20 años el basurero de la ciudad de México y de la zona conurbada, que abarcó más de 100 hectáreas y que recolectaba 2738 toneladas diarias de basura. Este basurero dejó de funcionar tres años antes de que esta investigación se realizara y actualmente es una zona habitacional. Hoy esta zona de basureros está situada en la periferia de Ciudad Netzahualcóyotl, Los Reyes La Paz, Chalco y el Lago de Texcoco.

No parece coincidencia la mayor concentración de casos de $\mathrm{NH}$ en esta zona, sino, más bien, parece existir algún factor común adicional en los pacientes y es posible que éste sea de tipo ambiental. En la figura 1 se observa la distribución aproximada de los casos de acuerdo con su localización y tiende a limitarse a una región específica. No fue el propósito de este trabajo buscar los probables agresores ambientales, sino establecer que esta asociación no es producto del azar. Sin embargo, podríamos especular que estas zonas que eran y las que aún son basureros albergan una gran cantidad de desechos orgánicos que pueden disecarse y quedar suspendidos en el aire ambiental. Estas partículas orgánicas podrían actuar como antígenos o coadyuvar al efecto del antígeno aviario y precipitar la enfermedad si fueran inhalados en forma continua por las personas susceptibles. En este orden de ideas, Rivera y colaboradores ${ }^{13}$ reportaron el aislamiento de diferentes cepas de amoebas de la atmósfera de la ciudad de México. Las muestras en ese estudio fueron tomadas entre 1 y $11 \mathrm{~m}$ de altura por arriba del suelo durante la temporada de lluvias en algunas zonas del sur del Distrito Federal. Los autores pudieron aislar tanto cepas conocidas patógenas como no patógenas en el humano. En otro trabajo, Rosas y colaboradores $^{14,15}$ reportaron el aislamiento de 11 especies de algas en la ciudad de México. Las implicaciones patológicas en los habitantes de este lugar sobre estos hallazgos no han sido establecidas. En este sentido, hasta donde sabemos, este es el primer trabajo que intenta establecer una asociación entre factores ambientales y la prevalencia de la $\mathrm{NH}$, asumiendo que la ZUOP refleja, de alguna forma, el ambiente en el que vive un paciente. Por lo tanto, es sencillo suponer que si estas partículas son respiradas por un individuo podrían desencadenar una serie de respuestas, que pueden variar desde reacciones de hipersensibilidad hasta infecciones directas del tracto respiratorio. La combinación de susceptibilidad a la exposición del antígeno aviario más la inhalación de partículas orgánicas dispersas en el aire que se respira podrían actuar en forma sinérgica para desencadenar el cuadro de inflamación que se observa en los pacientes con $\mathrm{NH}$.

La NH es una enfermedad que guarda una estrecha asociación con la inhalación de antígenos en el ambiente. Existe una lista amplia de diferentes antígenos asociados con la enfermedad. ${ }^{2}$ Sin embargo, hasta donde sabemos, este es el primer reporte que señala al ambiente que se respira como un coadyuvante y no como un factor causal. La observación es importante porque tiene implicaciones con el concepto de susceptibilidad. No todas las personas expuestas al antígeno causal de una NH desarrollan la enfermedad, solamente un grupo. Se ha asumido que esto obedece a diferencias individuales que hace que algunas personas sean más susceptibles que otras. ${ }^{7}$ Las observaciones de este trabajo hacen evidente que ese concepto de susceptibilidad podría ser en realidad un efecto coadyuvante del ambiente en el que las personas se desarrollan.

En conclusión, este estudio señala la asociación de vivir en las zonas referidas anteriormente y de padecer $\mathrm{NH}$, así también la posibilidad de que pudieran existir en el ambiente partículas respirables que actúen como factores promotores para el desarrollo de la $\mathrm{NH}$. Es motivo de otro trabajo detectar antígenos ambientales y, en forma más ambiciosa, probar su reactividad en el suero de individuos afectados. Si los resultados de este estudio se repitieran o confirmaran, en forma directa o indirecta, mediante otros abordajes metodológicos, se deberían considerar cuando menos dos recomendaciones de política en salud pública, a saber: a) limitar la convivencia con aves en algunas de las zonas geográficas descritas en este trabajo, y b) mejorar el ambiente de las mismas de modo que se reduzca la probabilidad de enfermedades crónicas e incapacitantes.

\section{Referencias}

1. Selman M, C hapela R, Salas J, Sansores R, C arrillo G, Pérez-Padilla R et al. Hypersensitivity pneumonitis. Clinical approach and an integral concept about its pathogenesis. A Mexican point of view. En: Selman M, Barrios $R$, ed. Interstitial Pulmonary D iseases. Selected topics. Boca Raton (FL):CRC Press,1991:171-195. 
2. Reynolds HY. Hypersensitivity pneumonitis: Symposium on Interstitial Lung Disease. C lin Chest Med 1982; 3:503-519.

3. Selman M, Chapela R, Raghu G. Hypersensitivity pneumonitis: Clinical manifestations, diagnosis, pathogenesis and therapeutic strategies. Semin Resp Med 1993;14:353-364.

4. Antoniades N H, Bravo M, Avila R, Galanopoulus T, N eville J, Selman M. Platelet-derived growth factor in idiopathic pulmonary fibrosis.J $\mathrm{Clin}$ Invest 1990;86:1055-1064.

5. Pérez-Padilla J, Salas J, C hapela R, Sánchez M, C arrillo G, Pérez-C havira $R$ et al. Mortality in Mexican patients with chronic pigeon breeder's lung compared with those with usual interstitial pneumonia. Am Rev Respir Dis 1993;148:49-53.

6. Fink JN . Hipersensitivity pneumonitis. Clin Chest Med 1992;13:303.

7. Selman M, Terán L, Mendoza A, C amarena A, Martínez-Cordero E, Lezama $M$ et al. Increase of HLA-DR7 in pigeon breeder's lung in a Mexican population. C lin Immunol Immunopathol 1987; 44(I):63.

8. Selman, M, Salas J, Pardo A, Pérez-Padilla, R. Pigeon breeder's disease:A paradigm for understanding hypersensitivity pneumonitis. Clin Pulm Med 1995;2(2):98-105.
9. Murao M,Tamura M, Kawai T, Hamada K. A clinical study of hipersensitivity pneumonitis in Japan. Jpn J Thorac D is 1980;18:373.

10. Ando M, Arima K,Yoneda R, Tamura M. Japanese summer-type hypersensitivity pneumonitis. Geographic distribution, home environment, and clinical characteristics of 621 cases. Am Rev Respir D is 1991;144:765.

11. Schluger, N W, Romer W N. Current approaches to the diagnosis of active pulmonary tuberculosis.Am J Respir Crit Care Med 1994;149: 264-267.

12. N ational Heart, Lung and Blood Institute. G uidelines for the diagnosis and management of asthma. N ational Asthma, Education Program. Expert Panel Report. J Allergy C lin Immunol 1991;88(3 Suppl): 425-434. 13. Rivera F, Roy-0 cotla G, Rosas I, Ramirez E, Bonilla P, Lares F.Amoebae isolated from the atmosphere of Mexico C ity. Environ Res 1987;42: 149-154.

14. Rosas I, Roy-0 cotla G, Mosiño P. Meteorological effects on variation of airborne algae in Mexico. Int J Biometeorol 1989;81: 1-7.

15. Rosas I, Roy-Acotla G, Mosiño P, Baez A, Rivera L. Abundance and heterogeneity of algae in the Mexico City atmosphere. Geofis Int 1989; 26:359-373. 